\title{
RECONSTRUCTION OF THE COLLISION KERNEL IN THE NONLINEAR BOLTZMANN EQUATION
}

\author{
RU-YU LAI, GUNTHER UHLMANN, AND YANG YANG
}

\begin{abstract}
We consider an inverse problem for the Boltzmann equation with nonlinear collision operator in dimensions $n \geq 2$. We show that the kinetic collision kernel can be uniquely determined from the incoming-to-outgoing mappings on the boundary of the domain provided that the kernel satisfies a monotonicity condition. Furthermore, a reconstruction formula is also derived. The key methodology is based on the higher-order linearization scheme to reduce a nonlinear equation into simpler linear equations by introducing multiple small parameters into the original equation.
\end{abstract}

\section{INTRODUCTION}

1.1. Motivation. Kinetic theory describes the dynamics of a large number of particles from a microscopic point of view. In particular, kinetic theory enjoys a lot of unique properties and demonstrates complicated mathematical features, which put it at a very important place for scientific studies. Applications of the kinetic theory include the dynamics of dilute charged particles, the semiconductor device, and space plasma physics [12, 24].

Kinetic equations model the evolution of a many-body particle system by means of a singleparticle distribution function. The collision operators are particularly crucial for approximating the underlying details of the many-body particle interactions. Among all collision operators, arguably the most well-known one is the Boltzmann collision operator that describes the binary particle interaction by a kinetic distribution $F=F(x, v)$ and takes the form

$$
Q(F, F)=\int_{\mathbb{R}^{3}} \int_{\mathbb{S}^{2}} q(\xi, \theta)\left[F\left(x, u^{\prime}\right) F\left(x, v^{\prime}\right)-F(x, u) F(x, v)\right] d \omega d u,
$$

where $\xi=|v-u|$ and $\cos \theta=(v-u) \cdot \omega /|v-u|, \omega \in \mathbb{S}^{2}$. The vectors

$$
u^{\prime}=u-[(u-v) \cdot \omega] \omega \text { and } v^{\prime}=v+[(u-v) \cdot \omega] \omega
$$

denote velocities after a collision of particles having velocities $v, u$ before the collision. The function $q(\xi, \theta)$ is called the collision cross section (kernel) and its form depends on the species of particles. For example, in the hard potential, the collision kernel is $q(\xi, \theta)=|v-u|^{\gamma} q_{0}\left(\frac{v-u}{|v-u|} \cdot \omega\right)$, where $0<\gamma \leq 1$ and $\int_{\mathbb{S}^{2}} q_{0}(\theta \cdot \omega) d \omega<\infty$ for $\theta \in \mathbb{S}^{2}$.

In the forward problem, there have been substantial contributions in the mathematical study of various aspects of Boltzmann equations. These involve the existence and uniqueness of the solutions, the decay of solutions toward a Maxwellian, as well as the connection between the kinetic theory and fluid dynamics, see for instance [18, 19, 21, 22, 23, 25, 28, 41, 45] and the references therein.

1.2. Inverse Problem. The inverse problem for kinetic equations is to find out hidden properties of the unknown parameters in the equations from the experimental data. Due to the importance of the collision operator in the kinetic theory, there is an increasing interest in solving these problems. The aim here is to study the identification of the unknown collision kernel from indirect measurements on the boundary.

Key words: Nonlinearity, Boltzmann equation, Inverse problems, Collision operator. 
Let us describe the Boltzmann equation studied in this article. Let $\Omega \subset \mathbb{R}^{n}$ be a bounded domain with $C^{\infty}$ boundary $\partial \Omega$ with $n \geq 2$. We consider the following Boltzmann equation:

$$
\begin{cases}v \cdot \nabla_{x} F=Q(F, F) & \text { in } \Omega \times \mathbb{R}^{n}, \\ F=g & \text { on } \Gamma_{-},\end{cases}
$$

where $F(x, v)$ is the distribution function that depends on the position $x \in \Omega$ and the velocity $v \in \mathbb{R}^{n}$. Throughout this paper, the collision operator takes the form

$$
Q\left(H_{1}, H_{2}\right)=\int_{\mathbb{R}^{n}} \int_{\mathbb{S}^{n-1}} B(v, u, \omega)\left[H_{1}\left(x, u^{\prime}\right) H_{2}\left(x, v^{\prime}\right)-H_{1}(x, u) H_{2}(x, v)\right] d \omega d u,
$$

where $B(v, u, \omega)$ is the collision kernel and $u^{\prime}, v^{\prime}$ are defined in (1.1).

We denote the boundary operator $\mathcal{A}$ that maps from the incoming data $F \in C\left(\Gamma_{-}\right)$on $\Gamma_{-}$to the outgoing one on $\Gamma_{+}$by

$$
\mathcal{A}:\left.\left.F\right|_{\Gamma_{-}} \mapsto F\right|_{\Gamma_{+}} \in C\left(\Gamma_{+}\right) .
$$

Here the sets $\Gamma_{ \pm}$are defined through

$$
\Gamma_{ \pm}:=\left\{(x, v) \in \partial \Omega \times \mathbb{R}^{n}: \pm n(x) \cdot v>0\right\}
$$

where $n(x)$ is the unit outer normal to $\partial \Omega$ at the point $x \in \partial \Omega$. It follows from Section 2 that the boundary value problem (1.2) is well-posed for small boundary data $g \in C\left(\Gamma_{-}\right)$. Hence, the map $\mathcal{A}$ is well-defined within the class of small boundary data. The inverse problem in this paper concerns the extraction of the information of the collision kernel $B$ from the incoming-to-outgoing boundary $\operatorname{map} \mathcal{A}$.

There have been related investigations in inverse problems for kinetic equations. One widely studied one is the radiative transfer equation, a linear Boltzmann equation with the linear collision operator. Let us introduce the problem for the RTE briefly below. The main objective is to determine the optical parameters from the albedo operator, that is known as the associated boundary operator to the RTE. In particular, the uniqueness and stability issues have been extensively addressed in the literature. In [10, 13, 14, 15, 16, 42, the parameters are uniquely determined from the boundary measurements. The key ingredient of such reconstruction mainly replies on the singular decomposition of the collision kernel that was developed in [14, 16]. In terms of the stability, Lipschitz stability estimates were studied in [3, 4, 5, 6, 32, 36, 46, 47]. Furthermore, this inverse problem for the RTE has also been investigated in the Riemannian setting, see for example [2, 17, 37, 38, 39, 40,

To study inverse problems for nonlinear equations, there is a classical method introduced by Isakov [26]. This method is to show that the boundary map for the nonlinear equation determines the analogue for its linearized equation. Then one can apply the existing result of inverse problems for such linearized equation to identify the unknown property. However this method does not work for the inverse problem considered here. Since the collision operator highly depends on the velocities before and after the collisions, the first linearization of (1.2) is fundamentally different from the RTE (a linear Boltzmann equation). As a result, the previously known theory for the RTE does not provide direct help to determine the kernel of (1.3).

In (1.2), the nonlinear interaction in the collision operator (1.3) introduces certain degree of difficulty to the investigation of the inverse problem. To overpass this difficulty, we introduce the higher-order linearization technique to the nonlinear Boltzmann equation. This technique employs nonlinearity as a tool in solving inverse problems for nonlinear equations. Its central idea is based on bringing in several small parameters into the data, and then differentiating the nonlinear equation with respect to these parameters to earn simpler linearized equations. In particular, the work [31] discovered that the nonlinearity can be beneficial in solving the inverse problem for the nonlinear hyperbolic equation, see also [11, 35] and the references therein. For the nonlinear elliptic equation, the works [7, 27, 43, 44 have studied the second order linearization of the nonlinear boundary map. 
Moreover, this method has been applied to study various inverse problems for elliptic equations with power-type nonlinearities in [1, 20, 29, 30, 33, 34.

When one applies the higher-order linearization to the problem (1.2), one can expect that the analysis of recovering the kernel will be very different from the case for the elliptic equations with nonlinearity, such as $\Delta u+q(x) u^{k}=0$ for a positive integer $k$ in [30, 33]. The difference not only comes from the type of equations, but also the form of nonlinearity. Compared to the nonlinearity $u^{k}(x)$ in the elliptic equations, the nonlinearities $F\left(x, u^{\prime}\right) F\left(x, v^{\prime}\right)$ and $F(x, u) F(x, v)$ here depend on different variables. Thus, the second linearized equation has more terms than pure power-type nonlinearity. Nevertheless, the unknown kernel only appears in the second linearization of (1.2) and leaves the first linearization of (1.2) plenty of freedom to choose its solutions. These turn out to be a crucial ingredient to determine the kernel. The detailed discussion is in Section 3 and Section 4 .

1.3. Main Results. Let $\Omega \subset \mathbb{R}^{n}$ be a bounded domain with $C^{\infty}$ boundary $\partial \Omega$ with $n \geq 2$. For $(x, v) \in \bar{\Omega} \times\left(\mathbb{R}^{n} \backslash\{0\}\right)$, we define $\tau_{ \pm}(x, v)$ as the exit time from the point $x$ to the boundary $\partial \Omega$ in the direction $\pm v$, namely,

$$
\tau_{ \pm}(x, v):=\sup \{s \geq 0: x \pm s v \in \Omega\} .
$$

Suppose that the kernel $B \in C\left(\mathbb{R}^{n} \times \mathbb{R}^{n} \times \mathbb{S}^{n-1}\right)$ satisfies the following condition: there exists a constant $M>0$ such that

$$
\tau_{ \pm}(x, v) \int_{\mathbb{R}^{n}} \int_{\mathbb{S}^{n-1}}|B(v, u, \omega)| d \omega d u<M<\infty
$$

for all $(x, v) \in \bar{\Omega} \times \mathbb{R}^{n}$. We now state the main results.

Theorem 1.1 (Monotonicity uniqueness). Let $\Omega \subset \mathbb{R}^{n}$ be a bounded domain with $C^{\infty}$ boundary $\partial \Omega$ with $n \geq 2$. Let the collision kernel $B_{j} \equiv B_{j}(v, u, \omega)$ be in $C\left(\mathbb{R}^{n} \times \mathbb{R}^{n} \times \mathbb{S}^{n-1}\right)$ and satisfy (1.5). Let $\mathcal{A}_{j}$ be the boundary operator of the problem (1.2) with the kernel $B$ replaced by $B_{j}$ for $j=1,2$. Suppose that

$$
\mathcal{A}_{1}(g)=\mathcal{A}_{2}(g)
$$

for all $g \in C\left(\Gamma_{-}\right)$with $\|g\|_{C\left(\Gamma_{-}\right)}<\varepsilon$, where $\varepsilon$ is a sufficiently small number. If $B_{1} \geq B_{2}$ pointwisely in $\mathbb{R}^{n} \times \mathbb{R}^{n} \times \mathbb{S}^{n-1}$ (Monotonicity condition), then

$$
B_{1}=B_{2} \text { everywhere in } \mathbb{R}^{n} \times \mathbb{R}^{n} \times \mathbb{S}^{n-1} .
$$

We also have the following reconstruction formula for $B$ provided that $B$ satisfies some weak assumptions.

Theorem 1.2 (Reconstruction formula). Let $\Omega \subset \mathbb{R}^{n}$ be a bounded domain with $C^{\infty}$ boundary $\partial \Omega$ with $n \geq 2$ and let $B \in C\left(\mathbb{R}^{n} \times \mathbb{R}^{n} \times \mathbb{S}^{n-1}\right)$ satisfy (1.5). Suppose that $B$ is symmetric in incoming velocities and is also an even function of $\omega$, that is,

$$
B(v, u, \omega)=B(u, v, \omega) \text { and } B(v, u,-\omega)=B(v, u, \omega) .
$$

Then for any $(a, b, \theta) \in \mathcal{D}$ (defined in (4.8)) in $\mathbb{R}^{n} \times \mathbb{R}^{n} \times \mathbb{S}^{n-1}$, we have

$$
\begin{aligned}
& S(a, a-[(a-b) \cdot \theta] \theta, b+[(a-b) \cdot \theta] \theta) \\
& =|(a-b) \cdot \theta|^{-2}\left(B(a, b, \theta)+\left(|a-b|^{2}-|(a-b) \cdot \theta|^{2}\right)^{-1} B\left(a, b, \widehat{P_{\theta^{\perp}}(a-b)}\right),\right.
\end{aligned}
$$

where we denote $\hat{z}:=\frac{z}{|z|} \in \mathbb{S}^{n-1}$ and $P_{\theta^{\perp}}(a-b):=(a-b)-[(a-b) \cdot \theta] \theta$, and the function $S$ (defined in (4.2) $)$ is determined by the boundary measurement $\mathcal{A}$ only.

Following immediately from Theorem 1.2, we obtain the uniqueness result if $B$ satisfies (1.5)-(1.6).

Corollary 1.3 (Uniqueness: two special cases). Suppose that two collision kernels $B_{1}$ and $B_{2}$ satisfy (1.5) and (1.6) and have identical boundary measurements. Then $B_{1}=B_{2}$ in the following two cases: 
(1) the collision kernel $B=B(v, u)$ is independent of $\omega$;

(2) the monotonicity condition is valid, such as $B_{1} \geq B_{2}$.

Remark 1.1. Compared to Theorem 1.1, the uniqueness result of Corollary 1.3 is constructive yet replies on an additional assumption (1.6) since it is a direct consequence from the reconstruction formula stated in Theorem 1.2.

We note that Theorem 1.1 illustrates the uniqueness result is valid if $B$ satisfies the monotonicity condition and its proof in Section 3 replies on the suitable chosen Gaussian-like solutions to the first linearized equation of (1.2). To demonstrate Theorem 1.2, the methodology is based on a similar strategy in the study of the RTE by applying the solution having the boundary data that are only concentrated on the incoming direction. Thus, the information of the kernel $B$ can be carried out from the propagation of these particles.

1.4. Outline. The paper is organized as follows. Section 2 is devoted to prove fundamental results, including the well-posedness of (1.2). They will play an important role in the study of the determination of the kernel. In Section 3, we detail the analysis of the higher-order linearization scheme and provide the proof of Theorem [1.1, Furthermore, the reconstruction formula in Theorem 1.2 is presented and proved in Section 4 as well as the uniqueness results in two special cases are discussed under the same hypothesis.

\section{Preliminaries}

In this section, we introduce the results that are essential for the investigation of the proposed inverse problem for (1.2). The main goal here is to establish the well-posedness for the boundary value problem (1.2) with small incoming boundary data.

We first discuss the following lemma as preparation for the well-posedness result.

Lemma 2.1. Suppose that $\sigma \in L^{\infty}(\Omega)$ satisfies $\sigma \geq \sigma_{0}>0$ for positive constant $\sigma_{0}$. For $f \in$ $C\left(\Omega \times \mathbb{R}^{n}\right)$ and $g \in C\left(\Gamma_{-}\right)$, the solution $F$ to

$$
\begin{cases}v \cdot \nabla_{x} F+\sigma F=f & \text { in } \Omega \times \mathbb{R}^{n} \\ F=g & \text { on } \Gamma_{-}\end{cases}
$$

has the form

$$
F(x, v)=e^{-\int_{0}^{\tau_{-}(x, v)} \sigma(x-s v) d s} g\left(x-\tau_{-}(x, v) v, v\right)+\int_{0}^{\tau_{-}(x, v)} e^{-\int_{0}^{s} \sigma(x-\eta v) d \eta} f(x-s v, v) d s
$$

and satisfies the estimate

$$
\|F\|_{C\left(\Omega \times \mathbb{R}^{n}\right)} \leq\|g\|_{C\left(\Gamma_{-}\right)}+C\|f\|_{C\left(\Omega \times \mathbb{R}^{n}\right)},
$$

where $C$ depends only on $\sigma_{0}$.

Proof. It can be readily verified that $F(x, v)$ defined above is indeed a solution. Moreover, from the representation of $F$ and $\sigma \geq \sigma_{0}>0$, we have

$$
\begin{aligned}
|F(x, v)| & =\left|e^{-\int_{0}^{\tau_{-}(x, v)} \sigma(x-s v) d s} g\left(x-\tau_{-}(x, v) v, v\right)+\int_{0}^{\tau_{-}(x, v)} e^{-\int_{0}^{s} \sigma(x-\eta v) d \eta} f(x-s v, v) d s\right| \\
& \leq\|g\|_{C\left(\Gamma_{-}\right)}+\|f\|_{C\left(\Omega \times \mathbb{R}^{n}\right)} \int_{0}^{\tau_{-}(x, v)} e^{-\sigma_{0} s} d s \\
& \leq\|g\|_{C\left(\Gamma_{-}\right)}+\frac{1}{\sigma_{0}}\|f\|_{C\left(\Omega \times \mathbb{R}^{n}\right)}\left(1-e^{-\sigma_{0} \tau_{-}(x, v)}\right)
\end{aligned}
$$

for any $(x, v) \in \Omega \times \mathbb{R}^{n}$. Thus, the estimate (2.2) holds. 
Remark 2.1. We note that when $\sigma=0$, the solution takes the form

$$
F(x, v)=g\left(x-\tau_{-}(x, v) v, v\right)+\int_{0}^{\tau_{-}(x, v)} f(x-s v, v) d s .
$$

Then it is clear that

$$
|F(x, v)| \leq\|g\|_{C\left(\Gamma_{-}\right)}+\left|\int_{0}^{\tau_{-}(x, v)} f(x-s v, v) d s\right| \text { for all } x \in \Omega, v \in \mathbb{R}^{n} .
$$

2.1. Well-posedness. We consider the in-flow boundary condition for the Boltzmann equation

$$
\begin{cases}v \cdot \nabla_{x} F=Q(F, F) & \text { in } \Omega \times \mathbb{R}^{n}, \\ F=g & \text { on } \Gamma_{-},\end{cases}
$$

where the collision operator $Q$ is defined as in (1.3).

We show the boundary value problem for (2.4) is well-posed for small boundary data.

Theorem 2.2 (Well-posedness of the Boltzmann equation). Let $\Omega \subset \mathbb{R}^{n}, n \geq 2$ be a bounded domain with $C^{\infty}$ boundary $\partial \Omega$. Suppose that $B$ satisfies (1.5). Then there exists $\varepsilon>0$ such that when

$$
g \in \mathcal{X}:=\left\{g \in C\left(\Gamma_{-}\right):\|g\|_{C\left(\Gamma_{-}\right)} \leq \varepsilon\right\},
$$

the boundary value problem (2.4) has a unique solution $F$. Moreover, there exists a constant $C>0$, independent of $g$, such that

$$
\|F\|_{C\left(\Omega \times \mathbb{R}^{n}\right)} \leq C\|g\|_{C\left(\Gamma_{-}\right)} .
$$

Proof. We utilize the contraction mapping principle to show the existence of solution to (2.4).

To this end, we first take any $g \in C\left(\Gamma_{-}\right)$satisfying $\|g\|_{C\left(\Gamma_{-}\right)} \leq \varepsilon$ with $\varepsilon$ to be determined later, then there exists a unique solution $F_{0}$ to the equation

$$
\begin{cases}v \cdot \nabla_{x} F_{0}=0 & \text { in } \Omega \times \mathbb{R}^{n} \\ F_{0}=g & \text { on } \Gamma_{-}\end{cases}
$$

and $F_{0}$ satisfies

$$
\left\|F_{0}\right\|_{C\left(\Omega \times \mathbb{R}^{n}\right)} \leq\|g\|_{C\left(\Gamma_{-}\right)} \leq \varepsilon .
$$

Second, if $F$ is the solution to (2.4), then we have that $G:=F-F_{0}$ satisfies

$$
\begin{cases}v \cdot \nabla_{x} G=Q\left(F_{0}+G, F_{0}+G\right)=: \mathcal{F}(G) & \text { in } \Omega \times \mathbb{R}^{n} \\ G=0 & \text { on } \Gamma_{-} .\end{cases}
$$

We denote by $\mathcal{L}^{-1}$ the solution operator to (2.8) and, moreover, from (2.3), it satisfies

$$
\left|\mathcal{L}^{-1}(\mathcal{F}(G))(x, v)\right| \leq C \tau_{-}(x, v)\|\mathcal{F}(G)(\cdot, v)\|_{C(\Omega)}
$$

for all $x \in \Omega$ and $v \in \mathbb{R}^{n}$.

Now we will show that $\mathcal{L}^{-1} \circ \mathcal{F}$ is a contraction map on a suitable subset of $C\left(\Omega \times \mathbb{R}^{n}\right)$. We first define the subspace $\mathfrak{X}$ of $C\left(\Omega \times \mathbb{R}^{n}\right)$ by

$$
\mathfrak{X}=\left\{\varphi \in C\left(\Omega \times \mathbb{R}^{n}\right):\left.\varphi\right|_{\Gamma_{-}}=0,\|\varphi\|_{C\left(\Omega \times \mathbb{R}^{n}\right)} \leq \delta\right\}
$$

with some constant $\delta>0$ to be determined later. To simplify the notation, we further define an operator $\mathcal{M}$ on $\mathfrak{X}$ by

$$
\mathcal{M}(\varphi)=\left(\mathcal{L}^{-1} \circ \mathcal{F}\right)(\varphi)
$$


for any $\varphi \in \mathfrak{X}$. From the direct computations, (2.7), and (2.9), we obtain

$$
\begin{aligned}
& |\mathcal{M}(\varphi)(x, v)| \\
& =\left|\mathcal{L}^{-1}\left(Q\left(F_{0}+\varphi, F_{0}+\varphi\right)\right)(x, v)\right| \\
& \leq C \tau_{-}(x, v)\left\|Q\left(F_{0}+\varphi, F_{0}+\varphi\right)(\cdot, v)\right\|_{C(\Omega)} \\
& =C \tau_{-}(x, v) \| \int_{\mathbb{R}^{n}} \int_{\mathbb{S}^{n-1}} B(v, u, w)\left[\left(F_{0}+\varphi\right)\left(x, u^{\prime}\right)\left(F_{0}+\varphi\right)\left(x, v^{\prime}\right)\right. \\
& \left.\quad-\left(F_{0}+\varphi\right)(x, u)\left(F_{0}+\varphi\right)(x, v)\right] d w d u \|_{C(\Omega)} \\
& \leq C \tau_{-}(x, v)\left(\int_{\mathbb{R}^{n}} \int_{\mathbb{S}^{n-1}}|B(v, u, w)| d w d u\right)(\varepsilon+\delta)^{2} \\
& \leq C M(\varepsilon+\delta)^{2} \text { for all }(x, v) \in \Omega \times \mathbb{R}^{n},
\end{aligned}
$$

where the last inequality is due to (1.5). Thus, we have

$$
\|\mathcal{M}(\varphi)\|_{C\left(\Omega \times \mathbb{R}^{n}\right)} \leq C M(\varepsilon+\delta)^{2} .
$$

Moreover, for any $\varphi_{1}, \varphi_{2} \in \mathfrak{X}$, we also estimate

$$
\begin{aligned}
& \mathcal{M}\left(\varphi_{1}\right)(x, v)-M\left(\varphi_{2}\right)(x, v) \mid \\
\leq & \tau_{-}(x, v)\left|\left(Q\left(F_{0}+\varphi_{1}, F_{0}+\varphi_{1}\right)(x, v)-Q\left(F_{0}+\varphi_{2}, F_{0}+\varphi_{2}\right)(x, v)\right)\right| \\
\leq & C \tau_{-}(x, v) \int_{\mathbb{R}^{n}} \int_{\mathbb{S}^{n-1}} B|(v, u, w)| d w d u\left(4\|F\|_{C\left(\Omega \times \mathbb{R}^{n}\right)}\left\|\varphi_{1}-\varphi_{2}\right\|_{C\left(\Omega \times \mathbb{R}^{n}\right)}\right. \\
& \left.+2\left\|\varphi_{1}\right\|_{C\left(\Omega \times \mathbb{R}^{n}\right)}\left\|\varphi_{1}-\varphi_{2}\right\|_{C\left(\Omega \times \mathbb{R}^{n}\right)}+2\left\|\varphi_{2}\right\|_{C\left(\Omega \times \mathbb{R}^{n}\right)}\left\|\varphi_{1}-\varphi_{2}\right\|_{C\left(\Omega \times \mathbb{R}^{n}\right)}\right) \\
\leq & C M(4 \varepsilon+4 \delta)\left\|\varphi_{1}-\varphi_{2}\right\|_{C\left(\Omega \times \mathbb{R}^{n}\right)} \text { for all }(x, v) \in \Omega \times \mathbb{R}^{n} .
\end{aligned}
$$

If we choose $1>\varepsilon>0$ and $\delta>0$ such that $\delta<\varepsilon$,

$$
C M(\varepsilon+\delta)^{2} \leq \delta
$$

and

$$
C M(4 \varepsilon+4 \delta)<1,
$$

then this leads to that $\mathcal{M}$ maps $\mathfrak{X}$ into itself and, moreover, is a contraction map on $\mathfrak{X}$. By the contraction mapping principle, there exists a unique fixed point $\hat{F} \in \mathfrak{X}$ of $\mathcal{M}$ such that $\hat{F}$ is the solution of (2.8). In particular, from (2.7) and (2.9), one can derive that

$$
\begin{aligned}
\|\hat{F}\|_{C\left(\Omega \times \mathbb{R}^{n}\right)} & \leq C M\left(\|g\|_{C\left(\Gamma_{-}\right)}^{2}+2\|g\|_{C\left(\Gamma_{-}\right)}\|\hat{F}\|_{C\left(\Omega \times \mathbb{R}^{n}\right)}+\|\hat{F}\|_{C\left(\Omega \times \mathbb{R}^{n}\right)}^{2}\right) \\
& \leq C M \varepsilon\|g\|_{C\left(\Gamma_{-}\right)}+C M(2 \varepsilon+\delta)\|\hat{F}\|_{C\left(\Omega \times \mathbb{R}^{n}\right)} .
\end{aligned}
$$

We further require that $\varepsilon$ and $\delta$ satisfy $2 \varepsilon+\delta \leq \gamma<1$ for some constant $\gamma$, we obtain

$$
\|\hat{F}\|_{C\left(\Omega \times \mathbb{R}^{n}\right)} \leq C\|g\|_{C\left(\Gamma_{-}\right)} .
$$

Finally, we conclude that $F=F_{0}+\hat{F}$ is a solution of (2.4) and satisfies the estimate

$$
\|F\|_{C\left(\Omega \times \mathbb{R}^{n}\right)} \leq\left\|F_{0}\right\|_{C\left(\Omega \times \mathbb{R}^{n}\right)}+\|\hat{F}\|_{C\left(\Omega \times \mathbb{R}^{n}\right)} \leq C\|g\|_{C\left(\Gamma_{-}\right)} .
$$

This completes the proof.

\section{Determination of the COlLision Kernel}

In this section, we will first perform the higher order linearization to the nonlinear Boltzmann equation. Under suitable constraints on the kernel, we will be able to uniquely determine the collision kernel from the boundary data. 
3.1. Linearization. Since the nonlinearity in (1.2) is quadratic-like, it is sufficiently to take parameters $\varepsilon=\left(\varepsilon_{1}, \varepsilon_{2}\right)$. For sufficiently small constants $\varepsilon_{1}, \varepsilon_{2}>0$ and $g_{1}, g_{2} \in C\left(\Gamma_{-}\right)$, by Theorem 2.2, there exists a unique solution $F=F(x, v ; \varepsilon)$ of the boundary value problem

$$
\begin{cases}v \cdot \nabla_{x} F=Q(F, F) & \text { in } \Omega \times \mathbb{R}^{n}, \\ F=\varepsilon_{1} g_{1}+\varepsilon_{2} g_{2} & \text { on } \Gamma_{-},\end{cases}
$$

and, specifically, the solution satisfies

$$
\|F\|_{C\left(\Omega \times \mathbb{R}^{n}\right)} \leq C \varepsilon_{1}\left\|g_{1}\right\|_{C\left(\Gamma_{-}\right)}+C \varepsilon_{2}\left\|g_{2}\right\|_{C\left(\Gamma_{-}\right)} .
$$

Next, let $V^{(k)}$ for $k=1,2$ be the solution of

$$
\begin{cases}v \cdot \nabla_{x} V^{(k)}=0 & \text { in } \Omega \times \mathbb{R}^{n}, \\ V^{(k)}=g_{k} & \text { on } \Gamma_{-},\end{cases}
$$

and then it satisfies

$$
\left\|V^{(k)}\right\|_{C\left(\Omega \times \mathbb{R}^{n}\right)} \leq C\left\|g_{k}\right\|_{C\left(\Gamma_{-}\right)} .
$$

Lastly, we consider $W$ to be the solution to the boundary value problem

$$
\begin{cases}v \cdot \nabla_{x} W=S(x, v) & \text { in } \Omega \times \mathbb{R}^{n}, \\ W=0 & \text { on } \Gamma_{-},\end{cases}
$$

where the function $S$ is denoted by

$$
\begin{aligned}
S(x, v):=\int_{\mathbb{R}^{n}} \int_{\mathbb{S}^{n-1}} B(v, u, \omega) & {\left[V^{(1)}\left(x, v^{\prime}\right) V^{(2)}\left(x, u^{\prime}\right)+V^{(1)}\left(x, u^{\prime}\right) V^{(2)}\left(x, v^{\prime}\right)\right.} \\
& \left.-V^{(1)}(x, u) V^{(2)}(x, v)-V^{(1)}(x, v) V^{(2)}(x, u)\right] d \omega d u .
\end{aligned}
$$

In the following lemma, we show that the quotient $F / \varepsilon_{k}$ converges to $V^{(k)}$ in Lemma 3.1 as well as we justify the approximation of the second derivatives of $F$ with respect to $\varepsilon$ in Lemma 3.2 .

Before starting the lemma, we denote the following functions:

$$
F(x, v)=F(x, v ; \varepsilon), \quad F^{(1)}(x, v)=F\left(x, v ; \varepsilon_{1}, 0\right), \quad F^{(2)}(x, v)=F\left(x, v ; 0, \varepsilon_{2}\right) .
$$

Lemma 3.1. Suppose that the assumptions in Theorem 2.2 hold, then we get

$$
\begin{aligned}
& \lim _{\varepsilon_{1} \rightarrow 0}\left\|\varepsilon_{1}^{-1} F^{(1)}-V^{(1)}\right\|_{C\left(\Omega \times \mathbb{R}^{n}\right)}=0, \\
& \lim _{\varepsilon_{2} \rightarrow 0}\left\|\varepsilon_{2}^{-1} F^{(2)}-V^{(2)}\right\|_{C\left(\Omega \times \mathbb{R}^{n}\right)}=0 .
\end{aligned}
$$

Similarly, we also have

$$
\lim _{\varepsilon \rightarrow 0}\left\|\varepsilon_{1}^{-1}\left(F-F^{(2)}\right)-V^{(1)}\right\|_{C\left(\Omega \times \mathbb{R}^{n}\right)}=0
$$

and

$$
\lim _{\varepsilon \rightarrow 0}\left\|\varepsilon_{2}^{-1}\left(F-F^{(1)}\right)-V^{(2)}\right\|_{C\left(\Omega \times \mathbb{R}^{n}\right)}=0 .
$$

Proof. We first consider the difference of (3.1) and (3.2) for $k=1$, then we have

$$
\begin{cases}v \cdot \nabla_{x}\left(\varepsilon_{1}^{-1} F-V^{(1)}\right)=\varepsilon_{1}^{-1} Q(F, F) & \text { in } \Omega \times \mathbb{R}^{n}, \\ \varepsilon_{1}^{-1} F-V^{(1)}=\varepsilon_{1}^{-1} \varepsilon_{2} g_{2} & \text { on } \Gamma_{-} .\end{cases}
$$


By Remark 2.1 and (1.5), we have

$$
\begin{aligned}
& \left\|\varepsilon_{1}^{-1} F-V^{(1)}\right\|_{C\left(\Omega \times \mathbb{R}^{n}\right)} \\
& \leq\left\|\varepsilon_{1}^{-1} \varepsilon_{2} g_{2}\right\|_{C\left(\Gamma_{-}\right)}+C\left\|\int_{0}^{\tau_{-}(x, v)} \varepsilon_{1}^{-1} Q(F, F)(x-s v, v) d s\right\|_{C\left(\Omega \times \mathbb{R}^{n}\right)} \\
& \leq\left\|\varepsilon_{1}^{-1} \varepsilon_{2} g_{2}\right\|_{C\left(\Gamma_{-}\right)}+C \varepsilon_{1}^{-1}\|F\|_{C\left(\Omega \times \mathbb{R}^{n}\right)}^{2} \max _{\Omega \times \mathbb{R}^{n}}\left(\tau_{-}(x, v) \int_{\mathbb{R}^{n}} \int_{\mathbb{S}^{n-1}}|B| d \omega d u\right) \\
& \leq\left\|\varepsilon_{1}^{-1} \varepsilon_{2} g_{2}\right\|_{C\left(\Gamma_{-}\right)}+C \varepsilon_{1}^{-1}\left(\varepsilon_{1}\left\|g_{1}\right\|_{C\left(\Gamma_{-}\right)}+\varepsilon_{2}\left\|g_{2}\right\|_{C\left(\Gamma_{-}\right)}\right)^{2} M .
\end{aligned}
$$

Let $\varepsilon_{2} \rightarrow 0$ and then we have

$$
\left\|\varepsilon_{1}^{-1} F\left(x, v ; \varepsilon_{1}, 0\right)-V^{(1)}\right\|_{C\left(\Omega \times \mathbb{R}^{n}\right)} \rightarrow 0 \quad \text { when } \varepsilon_{1} \rightarrow 0 .
$$

Similarly, for $k=2$, it also leads to

$$
\left\|\varepsilon_{2}^{-1} F\left(x, v ; 0, \varepsilon_{2}\right)-V^{(2)}\right\|_{C\left(\Omega \times \mathbb{R}^{n}\right)} \rightarrow 0 \quad \text { when } \varepsilon_{2} \rightarrow 0 .
$$

Further, to show the second limits, we note that $\varepsilon_{1}^{-1}\left(F-F^{(2)}\right)-V^{(1)}$ satisfies the problem

$$
\begin{cases}v \cdot \nabla_{x}\left(\varepsilon_{1}^{-1}\left(F-F^{(2)}\right)-V^{(1)}\right)=\varepsilon_{1}^{-1}\left(Q(F, F)-Q\left(F^{(2)}, F^{(2)}\right)\right) & \text { in } \Omega \times \mathbb{R}^{n}, \\ \varepsilon_{1}^{-1}\left(F-F^{(2)}\right)-V^{(1)}=0 & \text { on } \Gamma_{-} .\end{cases}
$$

By a direct computation and applying Remark 2.1 and (1.5) again, we obtain the following estimate

$$
\begin{aligned}
& \left\|\varepsilon_{1}^{-1}\left(F-F^{(2)}\right)-V^{(1)}\right\|_{C\left(\Omega \times \mathbb{R}^{n}\right)} \\
& \leq C M\left(\|F\|_{C\left(\Omega \times \mathbb{R}^{n}\right)}+\left\|F^{(2)}\right\|_{C\left(\Omega \times \mathbb{R}^{n}\right)}\right)\left(\left\|\varepsilon_{1}^{-1}\left(F-F^{(2)}\right)-V^{(1)}\right\|_{C\left(\Omega \times \mathbb{R}^{n}\right)}+\left\|V^{(1)}\right\|_{C\left(\Omega \times \mathbb{R}^{n}\right)}\right) .
\end{aligned}
$$

Thus, we get

$$
\left(1-C \varepsilon_{1}-C \varepsilon_{2}\right)\left\|\varepsilon_{1}^{-1}\left(F-F^{(2)}\right)-V^{(1)}\right\|_{C\left(\Omega \times \mathbb{R}^{n}\right)} \leq\left(C \varepsilon_{1}+C \varepsilon_{2}\right)\left\|V^{(1)}\right\|_{C\left(\Omega \times \mathbb{R}^{n}\right)},
$$

that goes to zero when $\varepsilon \rightarrow 0$. This completes the proof of (3.8).

Following a similar computation as above, we can obtain (3.9).

Lemma 3.2. Moreover, we obtain

$$
\lim _{\varepsilon \rightarrow 0}\left\|\left(\varepsilon_{1} \varepsilon_{2}\right)^{-1}\left(F-F^{(2)}-F^{(1)}\right)-W\right\|_{C\left(\Omega \times \mathbb{R}^{n}\right)}=0 .
$$

Proof. We denote the function

$$
\mathcal{G}:=\left(\varepsilon_{1} \varepsilon_{2}\right)^{-1}\left(F(x, v ; \varepsilon)-F^{(2)}(x, v)-F^{(1)}(x, v)\right)-W(x, v),
$$

then $\mathcal{G}$ satisfies

$$
\begin{cases}v \cdot \nabla_{x} \mathcal{G}=\mathcal{H} & \text { in } \Omega \times \mathbb{R}^{n}, \\ \mathcal{G}=0 & \text { on } \Gamma_{-},\end{cases}
$$

where we used the notations defined in (3.5) and we define

$$
\mathcal{H}:=\left(\varepsilon_{1} \varepsilon_{2}\right)^{-1}\left(Q(F, F)-Q\left(F^{(2)}, F^{(2)}\right)-Q\left(F^{(1)}, F^{(1)}\right)\right)-Q\left(V^{(1)}, V^{(2)}\right)-Q\left(V^{(2)}, V^{(1)}\right) .
$$

By using Remark 2.1 and (1.5) again, it leads to

$$
\|\mathcal{G}\|_{C\left(\Omega \times \mathbb{R}^{n}\right)} \leq C M\left\|\mathcal{H}_{1}\right\|_{C\left(\Omega \times \mathbb{R}^{n}\right)}+C M\left\|\mathcal{H}_{2}\right\|_{C\left(\Omega \times \mathbb{R}^{n}\right)},
$$

where

$$
\begin{aligned}
\mathcal{H}_{1}:= & \left(\varepsilon_{1} \varepsilon_{2}\right)^{-1}\left(F\left(x, u^{\prime}\right) F\left(x, v^{\prime}\right)-F^{(2)}\left(x, u^{\prime}\right) F^{(2)}\left(x, v^{\prime}\right)-F^{(1)}\left(x, u^{\prime}\right) F^{(1)}\left(x, v^{\prime}\right)\right) \\
& -V^{(1)}\left(x, u^{\prime}\right) V^{(2)}\left(x, v^{\prime}\right)-V^{(1)}\left(x, v^{\prime}\right) V^{(2)}\left(x, u^{\prime}\right),
\end{aligned}
$$


and

$$
\begin{aligned}
\mathcal{H}_{2}:= & \left(\varepsilon_{1} \varepsilon_{2}\right)^{-1}\left(F(x, u) F(x, v)-F^{(2)}(x, u) F^{(2)}(x, v)-F^{(1)}(x, u) F^{(1)}(x, v)\right) \\
& -V^{(1)}(x, u) V^{(2)}(x, v)-V^{(1)}(x, v) V^{(2)}(x, u) .
\end{aligned}
$$

We observe that

$$
\begin{aligned}
& \lim _{\varepsilon \rightarrow 0}\left(\varepsilon_{1} \varepsilon_{2}\right)^{-1}\left(F\left(x, u^{\prime}\right) F\left(x, v^{\prime}\right)-F^{(2)}\left(x, u^{\prime}\right) F^{(2)}\left(x, v^{\prime}\right)-F^{(1)}\left(x, u^{\prime}\right) F^{(1)}\left(x, v^{\prime}\right)\right) \\
= & \lim _{\varepsilon \rightarrow 0} \varepsilon_{1}^{-1}\left(F\left(x, u^{\prime}\right)-F^{(2)}\left(x, u^{\prime}\right)\right) \varepsilon_{2}^{-1}\left(F\left(x, v^{\prime}\right)-F^{(1)}\left(x, v^{\prime}\right)\right)-\left(\varepsilon_{1} \varepsilon_{2}\right)^{-1} F^{(2)}\left(x, u^{\prime}\right) F^{(1)}\left(x, v^{\prime}\right) \\
& +\left(\varepsilon_{1} \varepsilon_{2}\right)^{-1}\left[F^{(2)}\left(x, u^{\prime}\right)\left(F\left(x, v^{\prime}\right)-F^{(2)}\left(x, v^{\prime}\right)\right)+F^{(1)}\left(x, v^{\prime}\right)\left(F\left(x, u^{\prime}\right)-F^{(1)}\left(x, u^{\prime}\right)\right)\right] \\
= & V^{(1)}\left(x, u^{\prime}\right) V^{(2)}\left(x, v^{\prime}\right)-V^{(1)}\left(x, v^{\prime}\right) V^{(2)}\left(x, u^{\prime}\right) \\
& +V^{(1)}\left(x, v^{\prime}\right) V^{(2)}\left(x, u^{\prime}\right)+V^{(1)}\left(x, v^{\prime}\right) V^{(2)}\left(x, u^{\prime}\right) \\
(3.15)= & V^{(1)}\left(x, u^{\prime}\right) V^{(2)}\left(x, v^{\prime}\right)+V^{(1)}\left(x, v^{\prime}\right) V^{(2)}\left(x, u^{\prime}\right),
\end{aligned}
$$

where we applied Lemma 3.1, Therefore, we have

$$
\lim _{\varepsilon \rightarrow 0}\left\|\mathcal{H}_{1}\right\|_{C\left(\Omega \times \mathbb{R}^{n}\right)}=0 .
$$

Similarly, replacing $u^{\prime}, v^{\prime}$ by $u, v$ in (3.15), we obtain

$$
\begin{aligned}
& \lim _{\varepsilon \rightarrow 0}\left(\varepsilon_{1} \varepsilon_{2}\right)^{-1}\left(F(x, u) F(x, v)-F^{(2)}(x, u) F^{(2)}(x, v)-F^{(1)}(x, u) F^{(1)}(x, v)\right) \\
& =V^{(1)}(x, u) V^{(2)}(x, v)+V^{(1)}(x, v) V^{(2)}(x, u),
\end{aligned}
$$

and then we get

$$
\lim _{\varepsilon \rightarrow 0}\left\|\mathcal{H}_{1}\right\|_{C\left(\Omega \times \mathbb{R}^{n}\right)}=0 .
$$

Thus, combining the above two limits of $\mathcal{H}_{j}$, it clearly implies that the right-hand side of (3.14) approaches to zero as $\varepsilon$ goes to zero. This completes the proof.

From Lemma 3.1 and Lemma 3.2, now we can denote the solution $V^{(k)}$ for (3.2) by the first derivative of $F,\left.\partial_{\varepsilon_{k}} F\right|_{\varepsilon=0}$, that is,

$$
V^{(k)}=\left.\partial_{\varepsilon_{k}} F\right|_{\varepsilon=0} .
$$

In particular, the solution $V^{(k)}$ takes the form

$$
V^{(k)}(x, v)=g_{k}\left(x-\tau_{-}(x, v) v, v\right)
$$

for any $(x, v) \in \Omega \times \mathbb{R}^{3}$ for $k=1,2$.

Moreover, we can also denote the solution $W$ for (3.3) by the second derivative $\left.\partial_{\varepsilon_{1}} \partial_{\varepsilon_{2}} F\right|_{\varepsilon=0}$, that is,

$$
W=\left.\partial_{\varepsilon_{1}} \partial_{\varepsilon_{2}} F\right|_{\varepsilon=0} .
$$

In addition, the solution $W$ can be expressed as

$$
W(x, v)=\int_{0}^{\tau_{-}(x, v)} S(x-s v, v) d s,
$$

where $S$ is defined in (3.4). 
3.2. Linearization of the boundary map. We first extend the Boltzmann solution to the boundary $\Gamma_{+}$in Lemma 3.3 and then show the boundedness of the operator $\mathcal{A}$ in Proposition 3.4. Finally, we turn to illustrate the linearization of $\mathcal{A}$ in Lemma 3.5 .

In the following lemma, we show a trace theorem in the spirit of [8, 9, see also [10, 16].

Lemma 3.3. Let $F$ be the solution of (2.4). Suppose that $B$ satisfies (1.5). For all $(x, v) \in \Gamma_{+}$, the limit $F(x, v)=\lim _{t \downarrow 0} F(x-t v, v)$ exists and, moreover, $F \in C\left(\Gamma_{ \pm}\right)$.

Proof. Since $g \in C\left(\Gamma_{-}\right)$and (1.5), we have $F$ and $v \cdot \nabla_{x} F$ are bounded in $\Omega \times \mathbb{R}^{n}$. Suppose that $B$ satisfies (1.5). For any $(x, v) \in \Gamma_{+}$, by the fundamental theorem of calculus, we conclude

$$
F(x, v)=F(x-t v, v)+\int_{0}^{t} v \cdot \nabla_{x} F(x-s v, v) d s,
$$

We have

$$
|F(x, v)-F(x-t v, v)|=\left|\int_{0}^{t} v \cdot \nabla_{x} F(x-s v, v) d s\right| \leq t\left\|v \cdot \nabla_{x} F\right\|_{C\left(\Omega \times \mathbb{R}^{n}\right)} .
$$

This implies that $\left.F\right|_{\Gamma_{+}}$in (3.18) is well-defined and $F(x, v)=\lim _{t \downarrow 0} F(x-t v, v)$ for all $(x, v) \in \Gamma_{+}$.

Lemma 3.3 immediately implies the following result.

Proposition 3.4. The boundary operator $\mathcal{A}$ is a bounded map $\mathcal{A}: \mathcal{X} \rightarrow C\left(\Gamma_{+}\right)$, where $\mathcal{X}$ is defined in (2.5).

Thus, Lemma 3.2 and Lemma 3.3 lead to the following result right away.

Lemma 3.5. For sufficiently small constants $\varepsilon_{1}, \varepsilon_{2}>0$ and $g_{1}, g_{2} \in C\left(\Gamma_{-}\right)$, we have

$$
\lim _{\varepsilon \rightarrow 0}\left\|\left(\varepsilon_{1} \varepsilon_{2}\right)^{-1}\left(\mathcal{A}\left(\varepsilon_{1} g_{1}+\varepsilon_{2} g_{2}\right)-\mathcal{A}\left(\varepsilon_{2} g_{2}\right)-\mathcal{A}\left(\varepsilon_{1} g_{1}\right)\right)-W\right\|_{C\left(\Gamma_{+}\right)}=0 .
$$

Remark 3.1. Based on the definition (3.16) and Lemma 3.5, the outgoing boundary value $\left.W\right|_{\Gamma_{+}}$ can be reconstructed as

$$
\left.W\right|_{\Gamma_{+}}=\lim _{\varepsilon \rightarrow 0}\left(\varepsilon_{1} \varepsilon_{2}\right)^{-1}\left(\mathcal{A}\left(\varepsilon_{1} g_{1}+\varepsilon_{2} g_{2}\right)-\mathcal{A}\left(\varepsilon_{2} g_{2}\right)-\mathcal{A}\left(\varepsilon_{1} g_{1}\right)\right) .
$$

We obtain that if $\mathcal{A}_{1}(g)=\mathcal{A}_{2}(g)$ for all $g \in \mathcal{X}$, then the boundary operator $\mathcal{A}$ uniquely determines the function $\left.W\right|_{\Gamma_{+}}$.

3.3. Proof of Theorem 1.1. We are ready to prove the first uniqueness result in this paper.

Proof of Theorem 1.1. When $|\varepsilon|$ is sufficiently small, the boundary value problem

$$
\begin{cases}v \cdot \nabla_{x} F_{j}=Q_{j}\left(F_{j}, F_{j}\right) & \text { in } \Omega \times \mathbb{R}^{n}, \\ F_{j}=\varepsilon_{1} g_{1}+\varepsilon_{2} g_{2} & \text { on } \Gamma_{-},\end{cases}
$$

has a unique small solution $F_{j}=F_{j}(x, v ; \varepsilon) \in C\left(\Omega \times \mathbb{R}^{n}\right)$. For $j=1,2$, differentiating the above equation with respect to $\varepsilon_{k}$ and taking $\varepsilon=0$, the function $V^{(k)}=\left.\partial_{\varepsilon_{k}} F_{j}\right|_{\varepsilon=0}$ is the solution to the problem

$$
\begin{cases}v \cdot \nabla_{x} V^{(k)}=0 & \text { in } \Omega \times \mathbb{R}^{n} \\ V^{(k)}=g_{k} & \text { on } \Gamma_{-}\end{cases}
$$

In addition, we also have

$$
W_{j}=\left.\partial_{\varepsilon_{1}} \partial_{\varepsilon_{2}} F_{j}\right|_{\varepsilon=0}
$$


satisfying the problem

$$
\begin{cases}v \cdot \nabla_{x} W_{j}=S_{j}(x, v) & \text { in } \Omega \times \mathbb{R}^{n} \\ W_{j}=0 & \text { on } \Gamma_{-}\end{cases}
$$

where the source term is

$$
\begin{aligned}
S_{j}(x, v)=\int_{\mathbb{R}^{n}} \int_{\mathbb{S}^{n-1}} B_{j}(v, u, \omega)[ & V^{(1)}\left(x, v^{\prime}\right) V^{(2)}\left(x, u^{\prime}\right)+V^{(1)}\left(x, u^{\prime}\right) V^{(2)}\left(x, v^{\prime}\right) \\
& \left.-V^{(1)}(x, u) V^{(2)}(x, v)-V^{(1)}(x, v) V^{(2)}(x, u)\right] d \omega d u .
\end{aligned}
$$

In particular, the solution $W_{j}$ can be written as

$$
W_{j}(x, v)=\int_{0}^{\tau_{-}(x, v)} S_{j}(x-s v, v) d s .
$$

Since the maps $\mathcal{A}_{1}(g)=\mathcal{A}_{2}(g)$ for all boundary data $g \in \mathcal{X}$, from Remark 3.1, we have

$$
\left.W_{1}\right|_{\Gamma_{+}}=\left.W_{2}\right|_{\Gamma_{+}} .
$$

Thus, for any $(x, v) \in \Gamma_{+}$, one can derive that

$$
\begin{aligned}
0 & =\int_{0}^{\tau_{-}(x, v)}\left(S_{1}-S_{2}\right)(x-s v, v) d s \\
& =\int_{0}^{\tau_{-}(x, v)}\left(\int_{\mathbb{R}^{n}} \int_{\mathbb{S}^{n-1}}\left(B_{1}-B_{2}\right)(v, u, \omega) P(x-s v, v, u, \omega) d \omega d u\right) d s,
\end{aligned}
$$

where we denote $P$ by

$$
\begin{aligned}
P(x, v, u, \omega):=V^{(1)}\left(x, v^{\prime}\right) & V^{(2)}\left(x, u^{\prime}\right)+V^{(1)}\left(x, u^{\prime}\right) V^{(2)}\left(x, v^{\prime}\right) \\
& -V^{(1)}(x, u) V^{(2)}(x, v)-V^{(1)}(x, v) V^{(2)}(x, u) .
\end{aligned}
$$

Fixing a nonzero vector $v_{0} \in \mathbb{R}^{n}$. Note that the equation (3.20) is independent of the kernel. Thus, we can freely choose

$$
V^{(1)}(x, v)=e^{\left|v-v_{0}\right|^{2}} \text { and } V^{(2)}(x, v) \equiv 1 .
$$

By substituting them into (3.22), we obtain

$$
0=\int_{0}^{\tau_{-}(x, v)} \int_{\mathbb{R}^{n}} \int_{\mathbb{S}^{n-1}}\left(B_{1}-B_{2}\right)(v, u, \omega) P(v, u, \omega) d \omega d u d s,
$$

with

$$
P(v, u, \omega):=P(x, v, u, \omega)=e^{\left|u^{\prime}-v_{0}\right|^{2}}+e^{\left|v^{\prime}-v_{0}\right|^{2}}-e^{\left|v-v_{0}\right|^{2}}-e^{\left|u-v_{0}\right|^{2}} .
$$

By applying (1.1) with incoming velocities $u, v_{0}$, we obtain

$$
\left|u^{\prime}-v_{0}\right|^{2}=\left|u-v_{0}\right|^{2}-\left|\left(v_{0}-u\right) \cdot w\right|^{2} \text { and }\left|v^{\prime}-v_{0}\right|^{2}=\left|\left(v_{0}-u\right) \cdot \omega\right|^{2} \text {, }
$$

and then we apply these identities to derive that

$$
\begin{aligned}
P\left(v_{0}, u, \omega\right) & =e^{\left|u-v_{0}\right|^{2}-\left|\left(v_{0}-u\right) \cdot \omega\right|^{2}}+e^{\left|\left(v_{0}-u\right) \cdot \omega\right|^{2}}-1-e^{\left|u-v_{0}\right|^{2}} \\
& =\left(1-e^{-\left|\left(v_{0}-u\right) \cdot \omega\right|^{2}}\right)\left(e^{\left|\left(v_{0}-u\right) \cdot \omega\right|^{2}}-e^{\left|u-v_{0}\right|^{2}}\right) .
\end{aligned}
$$

We also denote the subspace $N_{v_{0} u}$ of the unit sphere by

$$
N_{v_{0} u}=\left\{z \in \mathbb{S}^{n-1}: z \perp\left(v_{0}-u\right) \text { or } z= \pm \frac{v_{0}-u}{\left|v_{0}-u\right|}\right\} .
$$


Then $P=0$ if and only if $\omega \in N_{v_{0} u}$. On the other hand, if $\omega \notin N_{v_{0} u}$, then $P\left(v_{0}, u, \omega\right)<0$. Thus, we have $P\left(v_{0}, u, \omega\right) \leq 0$. From the monotonicity condition $B_{1} \geq B_{2}$, we further get

$$
\left(B_{1}-B_{2}\right)\left(v_{0}, u, \omega\right) P\left(v_{0}, u, \omega\right) \leq 0 \text { for all } x \in \Omega, u \in \mathbb{R}^{n}, \omega \in \mathbb{S}^{n-1},
$$

which implies that

$$
0=\int_{0}^{\tau_{-}\left(x, v_{0}\right)} \int_{\mathbb{R}^{n}} \int_{\mathbb{S}^{n-1}}\left(B_{1}-B_{2}\right)\left(v_{0}, u, \omega\right) P\left(v_{0}, u, \omega\right) d \omega d u d s \leq 0 .
$$

Since $\left(B_{1}-B_{2}\right) P \leq 0$ and $B_{j}$ and $P$ are continuous, we have

$$
\left(B_{1}-B_{2}\right)\left(v_{0}, u, \omega\right) P\left(v_{0}, u, \omega\right)=0 \text { for all } u \in \mathbb{R}^{n}, \omega \in \mathbb{S}^{n-1} .
$$

Therefore, from the fact that $P<0$ for every $\omega \notin N_{v_{0} u}$ and the continuity of $B_{j}$, for any nonzero fixed vector $v_{0}$, we can conclude that $B_{1}\left(v_{0}, \cdot, \cdot\right)=B_{2}\left(v_{0}, \cdot, \cdot\right)$ in $\mathbb{R}^{n} \times \mathbb{S}^{n-1}$. Since $v_{0}$ is arbitrary, we can obtain $B_{1}=B_{2}$, which completes the proof.

\section{A Reconstruction formula}

In this section, we derive a reconstruction formula for the kernel $B$ by making use of special solutions $V^{(k)}$, that concentrate near the incoming directions. Furthermore, by using this established formula, we can show that the uniqueness results hold in two special cases, as stated in Corollary 1.3 .

Recall that for any $u, v \in \mathbb{R}^{n}$ and any $\omega \in \mathbb{S}^{n-1}$, we will insist two basic properties of the collision kernel $B$, as stated in (1.6):

(1) $B$ is symmetric in incoming velocities: $B(v, u, \omega)=B(u, v, \omega)$;

(2) $B$ is an even function of $\omega: B(v, u,-\omega)=B(v, u, \omega)$.

We recall that $W$ solves the boundary value problem (3.3), hence can be written as line integrals of the internal source $S$, see (3.17). We are interested in the case where $S$ does not depend on $x$. Then (3.17) reduces to

$$
W(x, v)=\tau_{-}(x, v) S(v)
$$

for $(x, v) \in \Gamma_{+}$. Therefore, we can recover $S(v)$ from $\mathcal{A}$ as long as there is at least one $x \in \partial \Omega$ such that $\tau_{-}(x, v) \neq 0$.

In view of (3.4), the way to make $S$ independent of $x$, as in the proof of Theorem 1.1, is to choose transport solutions $V^{(1)}$ and $V^{(2)}$ in (3.4) that only depend on $v$, that is,

$$
\begin{aligned}
S(v):=\int_{\mathbb{R}^{n}} \int_{\mathbb{S}^{n-1}} B(v, u, w)[ & V^{(1)}\left(v^{\prime}\right) V^{(2)}\left(u^{\prime}\right)+V^{(1)}\left(u^{\prime}\right) V^{(2)}\left(v^{\prime}\right) \\
& \left.-V^{(1)}(v) V^{(2)}(u)-V^{(1)}(u) V^{(2)}(v)\right] d w d u,
\end{aligned}
$$

where $V^{(k)}=V^{(k)}(v)$ automatically solves the transport equation $v \cdot \nabla_{x} V^{(k)}=0$ in $\Omega \times \mathbb{R}^{n}, k=1,2$. It suffices to construct special transport solutions to extract information on $B$. The $x$-independent solutions are sufficient for our purpose since $B=B(v, u, \omega)$ does not depend on $x$.

Pick three distinct vectors $u_{0}, v_{0}, v_{*} \in \mathbb{R}^{n}$. We formally choose $V^{(1)}=\delta_{v_{0}}, V^{(2)}=\delta_{u_{0}}$ in (4.2), then multiply (4.2) by the delta function $\delta_{v_{*}}(v)$ and integrate in $v$ over $\mathbb{R}^{n}$ to obtain

$$
S\left(v_{*}, v_{0}, u_{0}\right):=I_{1}+I_{2}+I_{3}+I_{4},
$$


where $I_{j}, j=1, \cdots, 4$, is defined by

$$
\begin{aligned}
I_{1} & :=\int_{\mathbb{R}^{n}} \int_{\mathbb{R}^{n}} \int_{\mathbb{S}^{n-1}} B(v, u, \omega) \delta_{v_{0}}\left(v^{\prime}\right) \delta_{u_{0}}\left(u^{\prime}\right) \delta_{v_{*}}(v) d \omega d u d v ; \\
I_{2} & :=\int_{\mathbb{R}^{n}} \int_{\mathbb{R}^{n}} \int_{\mathbb{S}^{n-1}} B(v, u, \omega) \delta_{v_{0}}\left(u^{\prime}\right) \delta_{u_{0}}\left(v^{\prime}\right) \delta_{v_{*}}(v) d \omega d u d v ; \\
I_{3} & :=-\int_{\mathbb{R}^{n}} \int_{\mathbb{R}^{n}} \int_{\mathbb{S}^{n-1}} B(v, u, \omega) \delta_{v_{0}}(v) \delta_{u_{0}}(u) \delta_{v_{*}}(v) d \omega d u d v ; \\
I_{4} & :=-\int_{\mathbb{R}^{n}} \int_{\mathbb{R}^{n}} \int_{\mathbb{S}^{n-1}} B(v, u, \omega) \delta_{v_{0}}(u) \delta_{u_{0}}(v) \delta_{v_{*}}(v) d \omega d u d v .
\end{aligned}
$$

The value $S\left(v_{*}, v_{0}, u_{0}\right)$ can be calculated from $\mathcal{A}$ using (4.1) and (3.19) with $g_{1}=\left.V^{(1)}\right|_{\Gamma_{-}}=\left.\delta_{v_{0}}\right|_{\Gamma_{-}}$ and $g_{2}=\left.V^{(2)}\right|_{\Gamma_{-}}=\left.\delta_{u_{0}}\right|_{\Gamma_{-}}$. We will divide the calculation of the four integrals into several lemmas and propositions. We note that the arguments below can be made rigorously by replacing the delta functions by limits of some smooth cut-off functions.

4.1. Preliminaries. We remark that $u^{\prime}$ and $v^{\prime}$ in the integrands of $I_{1}$ and $I_{2}$ should be interpreted as functions of $u$ and $v$, as was defined in (1.1). Since the map $(u, v) \mapsto\left(u^{\prime}, v^{\prime}\right)$ in (1.1) is an isometry for each $\omega \in \mathbb{S}^{n-1}$, one can invert it to write $(u, v)$ as functions of $\left(u^{\prime}, v^{\prime}\right)$ as well. Explicitly,

$$
u=u\left(u^{\prime}, v^{\prime}, \omega\right):=u^{\prime}-\left[\left(u^{\prime}-v^{\prime}\right) \cdot \omega\right] \omega, \quad v=v\left(u^{\prime}, v^{\prime}, \omega\right):=v^{\prime}+\left[\left(u^{\prime}-v^{\prime}\right) \cdot \omega\right] \omega .
$$

Some basic properties of these functions are recorded below.

Lemma 4.1. The functions $u=u\left(u^{\prime}, v^{\prime}, \omega\right)$ and $v=v\left(u^{\prime}, v^{\prime}, \omega\right)$ defined in (4.3) satisfy

(1) $u\left(u^{\prime}, v^{\prime},-\omega\right)=u\left(u^{\prime}, v^{\prime}, \omega\right)$ and $v\left(u^{\prime}, v^{\prime},-\omega\right)=v\left(u^{\prime}, v^{\prime}, \omega\right)$;

(2) $u\left(v^{\prime}, u^{\prime}, \omega\right)=v\left(u^{\prime}, v^{\prime}, \omega\right)$ and $v\left(v^{\prime}, u^{\prime}, \omega\right)=u\left(u^{\prime}, v^{\prime}, \omega\right)$.

Proof. These are straightforward calculations:

(1) $u\left(u^{\prime}, v^{\prime},-\omega\right)=u^{\prime}-\left[\left(u^{\prime}-v^{\prime}\right) \cdot(-\omega)\right](-\omega)=u^{\prime}-\left[\left(u^{\prime}-v^{\prime}\right) \cdot \omega\right] \omega=u\left(u^{\prime}, v^{\prime}, \omega\right)$. $v\left(u^{\prime}, v^{\prime},-\omega\right)=v^{\prime}+\left[\left(u^{\prime}-v^{\prime}\right) \cdot(-\omega)\right](-\omega)=v^{\prime}+\left[\left(u^{\prime}-v^{\prime}\right) \cdot \omega\right] \omega=v\left(u^{\prime}, v^{\prime}, \omega\right)$.

(2) $u\left(v^{\prime}, u^{\prime}, \omega\right)=v^{\prime}-\left[\left(v^{\prime}-u^{\prime}\right) \cdot \omega\right] \omega=v^{\prime}+\left[\left(u^{\prime}-v^{\prime}\right) \cdot \omega\right] \omega=v\left(u^{\prime}, v^{\prime}, \omega\right)$. $v\left(v^{\prime}, u^{\prime}, \omega\right)=u^{\prime}+\left[\left(v^{\prime}-u^{\prime}\right) \cdot \omega\right] \omega=u^{\prime}-\left[\left(u^{\prime}-v^{\prime}\right) \cdot \omega\right] \omega=u\left(u^{\prime}, v^{\prime}, \omega\right)$.

We study the solvability of two equations for $\omega$, which will be used later to compute $I_{1}$ and $I_{2}$. For any nonzero vector $u \in \mathbb{R}^{n}$, we denote by $\hat{u}$ the unit vector along the direction of $u$, that is, $\hat{u}:=\frac{u}{|u|} \in \mathbb{S}^{n-1}$.

Lemma 4.2. Let $u_{0}, v_{0}, v_{*} \in \mathbb{R}^{n}$ be three distinct vectors.

(1) The equation $v_{*}=v\left(u_{0}, v_{0}, \omega\right)$ admits solutions $\omega \in \mathbb{S}^{n-1}$ if and only if

$$
\left(v_{*}-v_{0}\right) \cdot\left(u_{0}-v_{0}\right)=\left|v_{*}-v_{0}\right|^{2} .
$$

When (4.4) holds, the solutions are $\omega= \pm \omega_{1}$, where $\omega_{1}:=\left(\widehat{v_{*}-v_{0}}\right)$.

(2) The equation $v_{*}=v\left(v_{0}, u_{0}, \omega\right)$ admits solutions $\omega \in \mathbb{S}^{n-1}$ if and only if

$$
-\left(v_{*}-u_{0}\right) \cdot\left(u_{0}-v_{0}\right)=\left|v_{*}-u_{0}\right|^{2} .
$$

When (4.5) holds, the solutions are $\omega= \pm \omega_{2}$, where $\omega_{2}:=\left(\widehat{v_{*}-u_{0}}\right)$.

Proof. First, in view of (4.3), the equation $v_{*}=v\left(u_{0}, v_{0}, \omega\right)$ is equivalent to

$$
v_{*}-v_{0}=\left[\left(u_{0}-v_{0}\right) \cdot \omega\right] \omega .
$$


If a solution $\omega \in \mathbb{S}^{n-1}$ exists, matching the directions implies $\omega= \pm \omega_{1}$, and matching the amplitudes implies

$$
\left|v_{*}-v_{0}\right|=\left|\left(u_{0}-v_{0}\right) \cdot \omega_{1}\right|=\left|\left(u_{0}-v_{0}\right) \cdot \frac{v_{*}-v_{0}}{\left|v_{*}-v_{0}\right|}\right|
$$

which is the desired relation (4.4). Conversely, if (4.4) holds, one has

$$
\left[\left(u_{0}-v_{0}\right) \cdot\left( \pm \omega_{1}\right)\right]\left( \pm \omega_{1}\right)=\left[\left(u_{0}-v_{0}\right) \cdot \frac{v_{*}-v_{0}}{\left|v_{*}-v_{0}\right|}\right] \frac{v_{*}-v_{0}}{\left|v_{*}-v_{0}\right|}=v_{*}-v_{0}
$$

indicating that $\omega= \pm \omega_{1}$ are solutions.

Second, switching the roles of $u_{0}$ and $v_{0}$ in (4.4) yields (4.5).

Next, we prove that the relations (4.4) and (4.5) are actually equivalent, and $\omega_{1}$ is orthogonal to $\omega_{2}$ whenever they exist. This is the content of the next lemma.

Lemma 4.3. Let $u_{0}, v_{0}, v_{*} \in \mathbb{R}^{n}$ be distinct vectors. Set $\omega_{1}:=\left(\widehat{v_{*}-v_{0}}\right)$ and $\omega_{2}:=\left(\widehat{v_{*}-u_{0}}\right)$. Then both (4.4) and (4.5) are equivalent to the orthogonality relation

$$
\left(v_{*}-v_{0}\right) \cdot\left(v_{*}-u_{0}\right)=0 .
$$

In particular, if one of $\omega_{1}$ and $\omega_{2}$ exists, so does the other, and we have $\omega_{1} \cdot \omega_{2}=0$.

Proof. The relation (4.4) is equivalent to

$$
\begin{aligned}
0 & =\left(v_{*}-v_{0}\right) \cdot\left(u_{0}-v_{0}\right)-\left|v_{*}-v_{0}\right|^{2}=\left(v_{*}-v_{0}\right) \cdot\left(u_{0}-v_{0}\right)-\left(v_{*}-v_{0}\right) \cdot\left(v_{*}-v_{0}\right) \\
& =\left(v_{*}-v_{0}\right) \cdot\left(u_{0}-v_{*}\right),
\end{aligned}
$$

which is (4.6). Switching the roles of $u_{0}$ and $v_{0}$ gives the equivalence of (4.5) and (4.6).

The solution $\omega_{1}$ exists if and only if (4.4) holds, which is equivalent to (4.6) thus (4.5). The latter holds if and only if $\omega_{2}$ exists. Finally, the directions of $\omega_{1}$ and $\omega_{2}$ are identical to those of $v_{*}-v_{0}$ and $v_{*}-u_{0}$, respectively, hence $\omega_{1} \cdot \omega_{2}=0$ whenever they exist.

Lemma 4.4. Let $u_{0}, v_{0}, v_{*} \in \mathbb{R}^{n}$ be distinct vectors and $\omega_{1}, \omega_{2}$ be defined as above. Suppose (4.6) holds so that $\omega_{1}$ and $\omega_{2}$ exist. We have

(1) $u\left(u_{0}, v_{0}, \omega_{2}\right)=v\left(u_{0}, v_{0}, \omega_{1}\right)=v_{*}$;

(2) $v\left(u_{0}, v_{0}, \omega_{2}\right)=u\left(u_{0}, v_{0}, \omega_{1}\right)=u_{0}+v_{0}-v_{*}$.

Proof. (1) We compute

$$
\begin{aligned}
u\left(u_{0}, v_{0}, \omega_{2}\right) & =u_{0}-\left[\left(u_{0}-v_{0}\right) \cdot \omega_{2}\right] \omega_{2}=u_{0}-\left[\left(u_{0}-v_{0}\right) \cdot \frac{v_{*}-u_{0}}{\left|v_{*}-u_{0}\right|}\right] \frac{v_{*}-u_{0}}{\left|v_{*}-u_{0}\right|} \\
& =u_{0}+v_{*}-u_{0}=v_{*},
\end{aligned}
$$

where the third equality comes from (4.5). On the other hand,

$$
\begin{aligned}
v\left(u_{0}, v_{0}, \omega_{1}\right) & =v_{0}+\left[\left(u_{0}-v_{0}\right) \cdot \omega_{1}\right] \omega_{1}=v_{0}+\left[\left(u_{0}-v_{0}\right) \cdot \frac{v_{*}-v_{0}}{\left|v_{*}-v_{0}\right|}\right] \frac{v_{*}-v_{0}}{\left|v_{*}-v_{0}\right|} \\
& =v_{0}+v_{*}-v_{0}=v_{*},
\end{aligned}
$$

where the third equality comes from (4.4).

(2) Likewise, we compute

$$
\begin{aligned}
v\left(u_{0}, v_{0}, \omega_{2}\right) & =v_{0}+\left[\left(u_{0}-v_{0}\right) \cdot \omega_{2}\right] \omega_{2}=v_{0}+\left[\left(u_{0}-v_{0}\right) \cdot \frac{v_{*}-u_{0}}{\left|v_{*}-u_{0}\right|}\right] \frac{v_{*}-u_{0}}{\left|v_{*}-u_{0}\right|} \\
& =v_{0}-\left(v_{*}-u_{0}\right)=v_{0}-v_{*}+u_{0}
\end{aligned}
$$


On the other hand,

$$
\begin{aligned}
u\left(u_{0}, v_{0}, \omega_{1}\right) & =u_{0}-\left[\left(u_{0}-v_{0}\right) \cdot \omega_{1}\right] \omega_{1}=u_{0}-\left[\left(u_{0}-v_{0}\right) \cdot \frac{v_{*}-v_{0}}{\left|v_{*}-v_{0}\right|}\right] \frac{v_{*}-v_{0}}{\left|v_{*}-v_{0}\right|} \\
& =u_{0}-\left(v_{*}-v_{0}\right)=u_{0}-v_{*}+v_{0} .
\end{aligned}
$$

4.2. Calculation of $I_{1}-I_{4}$. We are ready to compute the integrals $I_{k}, k=1, \cdots, 4$.

Proposition 4.5. Let $u_{0}, v_{0}, v_{*} \in \mathbb{R}^{n}$ be three distinct vectors, then

$$
\begin{aligned}
& I_{1}= \begin{cases}\left|\left(u_{0}-v_{0}\right) \cdot \omega_{1}\right|^{-n} B\left(v_{*}, u_{0}+v_{0}-v_{*}, \omega_{1}\right) & \text { if }(4.6) \text { holds } \\
0 & \text { otherwise }\end{cases} \\
& I_{2}= \begin{cases}\left|\left(u_{0}-v_{0}\right) \cdot \omega_{2}\right|^{-n} B\left(v_{*}, u_{0}+v_{0}-v_{*}, \omega_{2}\right) & \text { if (4.6) holds, } \\
0 & \text { otherwise. }\end{cases}
\end{aligned}
$$

Proof. We make the change of variable $(u, v) \mapsto\left(u^{\prime}, v^{\prime}\right)$ in $I_{1}$. The resulting Jacobian is 1 since the transformation is isometric for each $\omega \in \mathbb{S}^{n-1}$. Therefore,

$$
\begin{aligned}
I_{1} & =\int_{\mathbb{R}^{n}} \int_{\mathbb{R}^{n}} \int_{\mathbb{S}^{n-1}} B(v, u, \omega) \delta_{v_{0}}\left(v^{\prime}\right) \delta_{u_{0}}\left(u^{\prime}\right) \delta_{v_{*}}(v) d \omega d u d v \\
& =\int_{\mathbb{R}^{n}} \int_{\mathbb{R}^{n}} \int_{\mathbb{S}^{n-1}} B\left(v\left(u^{\prime}, v^{\prime}, \omega\right), u\left(u^{\prime}, v^{\prime}, \omega\right), \omega\right) \delta_{v_{0}}\left(v^{\prime}\right) \delta_{u_{0}}\left(u^{\prime}\right) \delta_{v_{*}}\left(v\left(u^{\prime}, v^{\prime}, \omega\right)\right) d \omega d u^{\prime} d v^{\prime} \\
& =\int_{\mathbb{S}^{n-1}} B\left(v\left(u_{0}, v_{0}, \omega\right), u\left(u_{0}, v_{0}, \omega\right), \omega\right) \delta_{v_{*}}\left(v\left(u_{0}, v_{0}, \omega\right)\right) d \omega .
\end{aligned}
$$

Thus, if $v_{*} \neq v\left(u_{0}, v_{0}, \omega\right)$, then $I_{1}=0$.

We have seen that the equation $v_{*}=v\left(u_{0}, v_{0}, \omega\right)$ have solutions $\omega= \pm \omega_{1}$ if and only if (4.6) holds. Therefore, combining with the change of variable, we can derive

$$
\begin{aligned}
I_{1} & =2^{-1}\left|\left(u_{0}-v_{0}\right) \cdot \omega_{1}\right|^{-n}\left(B\left(v\left(u_{0}, v_{0}, \omega_{1}\right), u\left(u_{0}, v_{0}, \omega_{1}\right), \omega_{1}\right)+B\left(v\left(u_{0}, v_{0},-\omega_{1}\right), u\left(u_{0}, v_{0},-\omega_{1}\right),-\omega_{1}\right)\right) \\
& =2^{-1}\left|\left(u_{0}-v_{0}\right) \cdot \omega_{1}\right|^{-n}\left(B\left(v\left(u_{0}, v_{0}, \omega_{1}\right), u\left(u_{0}, v_{0}, \omega_{1}\right), \omega_{1}\right)+B\left(v\left(u_{0}, v_{0}, \omega_{1}\right), u\left(u_{0}, v_{0}, \omega_{1}\right),-\omega_{1}\right)\right) \\
& =\left|\left(u_{0}-v_{0}\right) \cdot \omega_{1}\right|^{-n} B\left(v\left(u_{0}, v_{0}, \omega_{1}\right), u\left(u_{0}, v_{0}, \omega_{1}\right), \omega_{1}\right) \\
& =\left|\left(u_{0}-v_{0}\right) \cdot \omega_{1}\right|^{-n} B\left(v_{*}, u_{0}+v_{0}-v_{*}, \omega_{1}\right),
\end{aligned}
$$

where the term $2^{-1}\left|\left(u_{0}-v_{0}\right) \cdot \omega_{1}\right|^{-n}$ comes from the Jacobian, the second equality follows from Lemma 4.1, and the third equality is valid since $B(v, u, \omega)$ is assumed to be an even function of $\omega$, and the last equality follows from Lemma 4.4.

To obtain result (2), one just switches $u_{0}$ with $v_{0}$ in the above argument and applies the properties of $B$.

Proposition 4.6. Let $u_{0}, v_{0}, v_{*} \in \mathbb{R}^{n}$ be three distinct vectors. Then $I_{3}=I_{4}=0$.

Proof. $I_{3}$ can be computed as

$$
\begin{aligned}
I_{3} & =-\int_{\mathbb{R}^{n}} \int_{\mathbb{R}^{n}} \int_{\mathbb{S}^{n-1}} B(v, u, \omega) \delta_{v_{0}}(v) \delta_{u_{0}}(u) \delta_{v_{*}}(v) d \omega d u d v \\
& =-\int_{\mathbb{S}^{n-1}} B\left(v_{0}, u_{0}, \omega\right) \delta_{v_{*}}\left(v_{0}\right) d \omega \\
& =-\left(\int_{\mathbb{S}^{n-1}} B\left(v_{0}, u_{0}, \omega\right) d \omega\right) \delta_{v_{*}}\left(v_{0}\right)=0,
\end{aligned}
$$


where the last identity is valid since $v_{*}, v_{0}$ are distinct vectors.

Similarly, we have

$$
\begin{aligned}
I_{4} & =-\int_{\mathbb{R}^{n}} \int_{\mathbb{R}^{n}} \int_{\mathbb{S}^{n-1}} B(v, u, \omega) \delta_{v_{0}}(u) \delta_{u_{0}}(v) \delta_{v_{*}}(v) d \omega d u d v \\
& =-\int_{\mathbb{S}^{n-1}} B\left(u_{0}, v_{0}, \omega\right) \delta_{v_{*}}\left(u_{0}\right) d \omega \\
& =-\left(\int_{\mathbb{S}^{n-1}} B\left(v_{0}, u_{0}, \omega\right) d \omega\right) \delta_{v_{*}}\left(u_{0}\right)=0,
\end{aligned}
$$

where the last identity is also valid since $v_{*}, u_{0}$ are distinct vectors.

4.3. Recovery of $B$. We are ready to derive the reconstruction formula of the kernel $B$ in Theorem 1.2 when $B$ satisfies both conditions (1.5)-(1.6). In addition, with the established formula, we will discuss the uniqueness result under two different constraints as well as (1.5)-(1.6), while Theorem 1.1 only requires (1.5).

Proof of Theorem 1.2. Recall that

$$
S\left(v_{*}, v_{0}, u_{0}\right)=I_{1}+I_{2}+I_{3}+I_{4},
$$

where $I_{1}-I_{4}$ have been computed in Proposition 4.5 and Proposition 4.6.

Since $u_{0}, v_{0}, v_{*}$ are distinct, $I_{3}$ and $I_{4}$ vanish. We have, from Proposition 4.5, that

$$
\begin{aligned}
S\left(v_{*}, v_{0}, u_{0}\right) & =I_{1}+I_{2} \\
& = \begin{cases}\sum_{k=1}^{2}\left|\left(u_{0}-v_{0}\right) \cdot \omega_{k}\right|^{-n} B\left(v_{*}, u_{0}+v_{0}-v_{*}, \omega_{k}\right) & \text { if (4.6) holds, } \\
0 & \text { otherwise. }\end{cases}
\end{aligned}
$$

Given any $(a, b, \theta) \in \mathbb{R}^{n} \times \mathbb{R}^{n} \times \mathbb{S}^{n-1}$, we can choose

$$
v_{*}=a, \quad v_{0}=a-[(a-b) \cdot \theta] \theta, \quad u_{0}=b+[(a-b) \cdot \theta] \theta .
$$

Then

$$
u_{0}+v_{0}-v_{*}=b, \quad \omega_{1}= \pm \theta .
$$

One can check (4.6) holds for such triple $\left(v_{*}, v_{0}, u_{0}\right)$. Moreover, $v_{*}-u_{0}=(a-b)-[(a-b) \cdot \theta] \theta$ hence

where

$$
\omega_{2}=\left(\widehat{v_{*}-u_{0}}\right)=\widehat{P_{\theta^{\perp}}(a-b)},
$$

In addition, we also have

$$
P_{\theta^{\perp}}(a-b):=(a-b)-[(a-b) \cdot \theta] \theta .
$$

$$
\left|\left(u_{0}-v_{0}\right) \cdot \omega_{1}\right|^{2}=|(a-b) \cdot \theta|^{2}, \quad\left|\left(u_{0}-v_{0}\right) \cdot \omega_{2}\right|^{2}=|a-b|^{2}-|(a-b) \cdot \theta|^{2} .
$$

Therefore, we get

$$
\begin{aligned}
& S(a, a-[(a-b) \cdot \theta] \theta, b+[(a-b) \cdot \theta] \theta) \\
& =\left|\left(u_{0}-v_{0}\right) \cdot \omega_{1}\right|^{-2} B(a, b, \theta)+\left|\left(u_{0}-v_{0}\right) \cdot \omega_{2}\right|^{-2} B\left(a, b, \widehat{P_{\theta^{\perp}}(a-b)}\right) \\
& =|(a-b) \cdot \theta|^{-2}\left(B(a, b, \theta)+\left(|a-b|^{2}-|(a-b) \cdot \theta|^{2}\right)^{-1} B\left(a, b, \widehat{P_{\theta^{\perp}}(a-b)}\right),\right.
\end{aligned}
$$

which means we can recover the sum on the right hand side. The condition that $v_{*}, u_{0}, v_{0}$ being distinct translates to $(a, b, \theta)$ as

$$
\mathcal{D}=\left\{(a, b, \theta) \in \mathbb{R}^{n} \times \mathbb{R}^{n} \times \mathbb{S}^{n-1}:(a-b) \cdot \theta \neq 0 \text { and } a-b \neq[(a-b) \cdot \theta] \theta\right.
$$

$$
\text { and } a-b \neq 2[(a-b) \cdot \theta] \theta\} \text {. }
$$


As the complement of this set in $\mathbb{R}^{n} \times \mathbb{R}^{n} \times \mathbb{S}^{n-1}$ has Lebesgue measure zero, the formula (4.7) reconstructs the sum almost everywhere, and eventually everywhere if $B$ is continuous.

This reconstruction formula immediately leads to the unique determination of the kernel. Let's recall the statement of Corollary 1.3. Suppose that two collision kernels $B_{1}$ and $B_{2}$ have identical measurement. Then $B_{1}=B_{2}$ in the following two cases: (1) the collision kernel $B=B(v, u)$ is independent of $\omega ;(2)$ the monotonicity condition is valid, such as $B_{1} \geq B_{2}$.

Proof of Corollary 1.3. (1) Since $B$ is independent of $\omega$, we get

$$
B(a, b)=B(a, b, \theta)=B\left(a, b, \widehat{P_{\theta^{\perp}}(a-b)}\right)
$$

and thus (4.7) uniquely recovers $B$.

(2) Since $B_{1}$ and $B_{2}$ have the same boundary measurement, from (4.7), we have

$$
0=\left|\left(u_{0}-v_{0}\right) \cdot \omega_{1}\right|^{-n}\left(B_{1}-B_{2}\right)(a, b, \theta)+\left|\left(u_{0}-v_{0}\right) \cdot \omega_{2}\right|^{-2}\left(B_{1}-B_{2}\right)\left(a, b, \widehat{P_{\theta^{\perp}}(a-b)}\right) .
$$

This forces each term on the right hand side to vanish if $\left(u_{0}-v_{0}\right) \cdot \omega_{k} \neq 0, k=1,2$ since they are non-negative.

Acknowledgment. The project was initiated during the IMA workshop "Mathematics in Optical Imaging" in Spring 2019. The authors would like to thank the hospitality of the IMA. R.-Y. Lai is partially supported by the NSF grant DMS-1714490. G. Uhlmann is supported in part by the NSF, the Walker Family Endowed Professorship at the University of Washington, and a Si-Yuan Professorship at IAS, HKUST. Y. Yang is partially supported by the NSF grant DMS-1715178, AMS-Simons travel grant, and the start-up fund from Michigan State University.

\section{REFERENCES}

[1] Y. Assylbekov and T. Zhou. Direct and inverse problems for the nonlinear time-harmonic Maxwell equations in kerr-type media. Arxiv: 1709.07767, To appear in Journal of Spectral Theory, 2017.

[2] Y. M. Assylbekov and Y. Yang. An inverse radiative transfer in refractive media equipped with a magnetic field. The Journal of Geometric Analysis, 25:2148-2184, 2015.

[3] G. Bal and A. Jollivet. Stability estimates in stationary inverse transport. Inverse problems and Imaging, 2:427454, 2008.

[4] G. Bal and A. Jollivet. Stability estimates for time-dependent inverse transport. SIAM J. Math. Anal., 42(2):679700, 2010.

[5] G. Bal and A. Jollivet. Generalized stability estimates in inverse transport theory. Inverse problems and Imaging, 12(1):59-90, 2018.

[6] G. Bal and F. Monard. Inverse transport with isotropic time-harmonic sources. SIAM Journal on Mathematical Analysis, 44(1):134-161, 2012.

[7] C. Cârstea, G. Nakamura, and M. Vashisth. Reconstruction for the coefficients of a quasilinear elliptic partial differential equation. arXiv:1903.07034, 2019.

[8] M. Cessenat. Théorèmes de trace $\mathrm{L}^{p}$ pour des espaces de fonctions de la neutronique. C. R. Acad. Sci. Paris, Série I, 299:831-834, 1984.

[9] M. Cessenat. Théorèmes de trace pour des espaces de fonctions de la neutronique. C. R. Acad. Sci. Paris, Série I, 300:89-92, 1985.

[10] I.-K. Chen and D. Kawagoe. Propagation of boundary-induced discontinuity in stationary radiative transfer and its application to the optical tomography. Inverse Problems and Imaging, 13(2):337-351, 2019.

[11] X. Chen, M. Lassas, L. Oksanen, and G. Paternain. Detection of Hermitian connections in wave equations with cubic non-linearity. arXiv:1902.05711, 2019.

[12] Y. Cheng, I. M. Gamba, and K. Ren. Recovering doping profiles in semiconductor devices with the BoltzmannPoisson model. Journal of Computational Physics, 230:3391-3412, 2011.

[13] M. Choulli and P. Stefanov. Scattering inverse pour l'équation du transport et relations entre les opérateurs de scattering et d'albédo. C. R. Acad. Sci. Paris, 320:947-952, 1995. 
[14] M. Choulli and P. Stefanov. Inverse scattering and inverse boundary value problems for the linear Boltzmann equation. Comm. P.D.E., 21:763-785, 1996.

[15] M. Choulli and P. Stefanov. Reconstruction of the coefficients of the stationary transport equation from boundary measurements. Inverse Problems, 12:L19-L23, 1996.

[16] M. Choulli and P. Stefanov. An inverse boundary value problem for the stationary transport equation. Osaka J. Math., 36:87-104, 1998.

[17] N. S. Dairbekov, G. Paternain, P. Stefanov, and G. Uhlmann. The boundary rigidity problem in the presence of a magnetic field. Adv. Math., 216:535-609, 2007.

[18] L. Desvillettes and C. Villani. On the trend to global equilibrium for spatially inhomogeneous kinetic systems: The Boltzmann equation. Inventiones Mathematicae, 159:p.245316, 2005.

[19] R. J. DiPerna and P. L. Lions. On the Cauchy problem for Boltzmann equations: Global existence and weak stability. Annals of Mathematics, 130:321-366, 1989.

[20] A. Feizmohammadi and L. Oksanen. An inverse problem for a semi-linear elliptic equation in Riemannian geometries. arXiv:1904.00608, 2019.

[21] F. Golse and L. Saint-Raymond. The Navier-Stokes limit of the Boltzmann equation for bounded collision kernels. Inventiones Mathematicae, 155:81-161, 2004.

[22] F. Golse and L. Saint-Raymond. The incompressible Navier Sstokes limit of the Boltzmann equation for hard cutoff potentials. Journal de Mathematiques Pures et Appliquees, 91:508-552, 2009.

[23] Y. Guo. Decay and continuity of the Boltzmann equation in bounded domains. Arch. Rational Mech. Anal., 197:713-809, 2010.

[24] G. G. Howes. A prospectus on kinetic heliophysics. Phys. Plasmas, 24:055907, 2017.

[25] R. Illner and M. Shinbrot. The Boltzmann equation: Global existence for a rare gas in an infinite vacuum. Communications in Mathematical Physics, 95:217-226, 1984.

[26] V. Isakov. On uniqueness in inverse problems for semilinear parabolic equations. Archive for Rational Mechanics and Analysis, 124(1):1-12, 1993.

[27] H. Kang and G. Nakamura. Identification of nonlinearity in a conductivity equation via the Dirichlet-to-Neumann map. Inverse Problems, 18:1079-1088, 2002.

[28] C. Kim. Formation and propagation of discontinuity for Boltzmann equation in non-convex domains. Commun. Math. Phys., 308:641-701, 2011.

[29] K. Krupchyk and G. Uhlmann. Partial data inverse problems for semilinear elliptic equations with gradient nonlinearities. arXiv:1909.08122v1, 2019.

[30] K. Krupchyk and G. Uhlmann. A remark on partial data inverse problems for semilinear elliptic equations. To appear in Proceedings of the AMS, 2019.

[31] Y. Kurylev, M. Lassas, and G. Uhlmann. Inverse problems for Lorentzian manifolds and non-linear hyperbolic equations. Invent. Math., 212(3):781-857, 2018.

[32] R.-Y. Lai, Q. Li, and G. Uhlmann. Inverse problems for the stationary transport equation in the diffusion scaling. SIAM J. Appl. Math., 79(6):2340-2358, 2019.

[33] M. Lassas, T. Liimatainen, Y.-H. Lin, and M. Salo. Inverse problems for elliptic equations with power type nonlinearities. arXiv:1903.12562, 2019.

[34] M. Lassas, T. Liimatainen, Y.-H. Lin, and M. Salo. Partial data inverse problems and simultaneous recovery of boundary and coefficients for semilinear elliptic equations. arXiv:1905.02764, 2019.

[35] M. Lassas, G. Uhlmann, and Y. Wang. Inverse problems for semilinear wave equations on Lorentzian manifolds. Comm. Math. Phys., 360(2):555-609, 2018.

[36] M. Machida and M. Yamamoto. Global Lipschitz stability in determining coefficients of the radiative transport equation. Inverse Problems, 30:035010, 2014.

[37] S. McDowall, P. Stefanov, and A. Tamasan. Gauge equivalence in stationary radiative transport through media with varying index of refraction. Inverse Probl. Imaging, 4:151-167, 2010.

[38] S. McDowall, P. Stefanov, and A. Tamasan. Stability of the gauge equivalent in stationary inverse transport. Inverse Problems, 26:025006, 2010.

[39] S. McDowall, P. Stefanov, and A. Tamasan. Stability of the gauge equivalent classes in inverse stationary transport in refractive media. Contemp. Math., 559:85100, 2011.

[40] S. R. McDowall. An inverse problem for the transport equation in the presence of a Riemannian metric. Pacific journal of mathematics, 216:303-326, 2004.

[41] S. Mischler and B. Wennberg. On the spatially homogeneous Boltzmann equation. Annales de lInstitut Henri Poincare (C) Non Linear Analysis, 16:467-501, 1999.

[42] P. Stefanov and G. Uhlmann. Optical tomography in two dimensions. Methods Appl. Anal., 10:1-9, 2003.

[43] Z. Sun. On a quasilinear inverse boundary value problem. Math. Z., 221(2):293-305, 1996. 
RECONSTRUCTION OF THE COLLISION KERNEL IN THE NONLINEAR BOLTZMANN EQUATION

[44] Z. Sun and G. Uhlmann. Inverse problems in quasilinear anisotropic media. Amer. J. Math., 110(4):771-797, 1997.

[45] C. Villani. A Review of Mathematical Topics in Collisional Kinetic Theory, volume 1. North-Holland, 2002.

[46] J.-N. Wang. Stability estimates of an inverse problem for the stationary transport equation. Ann. Inst. H. Poincaré Phys. Théor., 70(5):473-495, 1999.

[47] H. Zhao and Y. Zhong. Instability of an inverse problem for the stationary radiative transport near the diffusion limit. SIAM J. Math. Anal., 51(5):3750-3768, 2019.

School of Mathematics, University of Minnesota, Minneapolis, MN 55455, USA

E-mail address: rylai@umn.edu

Department of Mathematics, University of Washington, Seattle, WA 98195, USA

HKuSt Jockey Club Institute for Advanced Study, HKUSt, Clear Water Bay, Kowloon, Hong KONG.

E-mail address: gunther@math.washington.edu

Department of Computational Mathematics Science and Engineering, Michigan State University, EAst LANsing, MI 48824, USA

E-mail address: yangy5@msu.edu 